\title{
Influence de la cavitation sur les caractéristiques moyennes et instantanées des turbines et des pompes-turbines
}

\section{Influence of cavitation on average and instantaneous characteristics of turbines and pump turbines}

\author{
G. Borciani
}

Directeur du Développement et de la Recherche,

Hydroart S.p.A. Milan

\section{R. Thalmann}

Chef du Service Hydraulique, Ateliers de Vevey

\section{Notations}

$\begin{array}{ll}D & (\mathrm{~m}) \\ g & \left(\mathrm{~m} / \mathrm{s}^{2}\right) \\ \rho & \left(\mathrm{kg} / \mathrm{m}^{3}\right) \\ Q & \left(\mathrm{~m}^{3} / \mathrm{s}\right)\end{array}$

$Q \quad\left(\mathrm{~m}^{3} / \mathrm{s}\right)$

$(\mathrm{gH}) \quad\left(\mathrm{J} / \mathrm{kg} ; \mathrm{m}^{2} / \mathrm{s}^{2}\right)$ énergie spécifique totale

$g(N P S H)\left(\mathrm{J} / \mathrm{kg} ; \mathrm{m}^{2} / \mathrm{s}^{2}\right)$ énergie spécifique nette positive à l'aspiration

$\begin{array}{lll}\omega & (\mathrm{rad} / \mathrm{s}) & \text { vitesse de rotation de la roue } \\ M & (\mathrm{Nm}) & \text { couple à la roue } \\ P & (\mathrm{~kW}) & \text { puissance à la roue }\end{array}$

$\varphi=\frac{(g H)}{\frac{1}{2}\left(\frac{\omega D}{2}\right)^{2}}$

$\varphi=\frac{Q}{\frac{\pi}{4} D^{2}\left(\omega \frac{D}{2}\right)}$

$\lambda=\frac{2(1000 \mathrm{P})}{\frac{\pi}{4} D^{2}\left(\frac{\omega D}{2}\right)^{3} \rho}$

$\sigma_{u}=\frac{g(N P S H)}{\frac{1}{2}\left(\frac{\omega D}{2}\right)^{2}}$

coefficient de cavitation

$k_{u}=\frac{\omega \frac{D}{2}}{\sqrt{2(g H)}}$

coefficient dc débit
$K_{Q}=\frac{Q}{\frac{\pi D^{2}}{4} \sqrt{2(g H)}} \quad$ coefficient de débit

$K_{M}=\frac{M}{\frac{\pi}{4} D^{2} \rho(g H) \frac{D}{2}} \quad$ coefficient de couple

$\sigma=\frac{g(N P S H)}{(g H)} \quad$ coefficient de cavitation

$\eta=\frac{P}{(\rho Q)(g H)} \quad$ rendement en turbine

$\eta=\frac{(\rho Q)(g H)}{P} \quad$ rendement en pompe

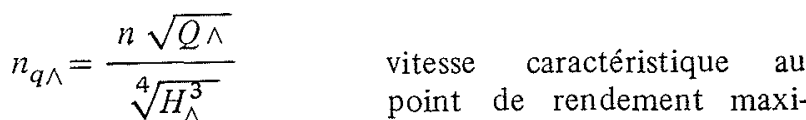
mum.

\section{Introduction}

L'influence de la cavitation sur le fonctionnement à débit partiel des turbines et pompes-turbines est étudiée sur la base d'expériences de laboratoire. Cette recherche examine les champs en dehors du fonctionnement normal, c'est-à-dire non seulement les champs à débit partiel (débit nul inclus), soit en turbine, soit en pompe, mais aussi ceux qui intéressent les transitoires : emballement, suremballement et hyperemballement. Sur la figure 1, les zones ay ant un intérêt particulier sont indiquées par des flèches. On trouve

LA HOUILLE BLANCHE/N² 2/3-1982 


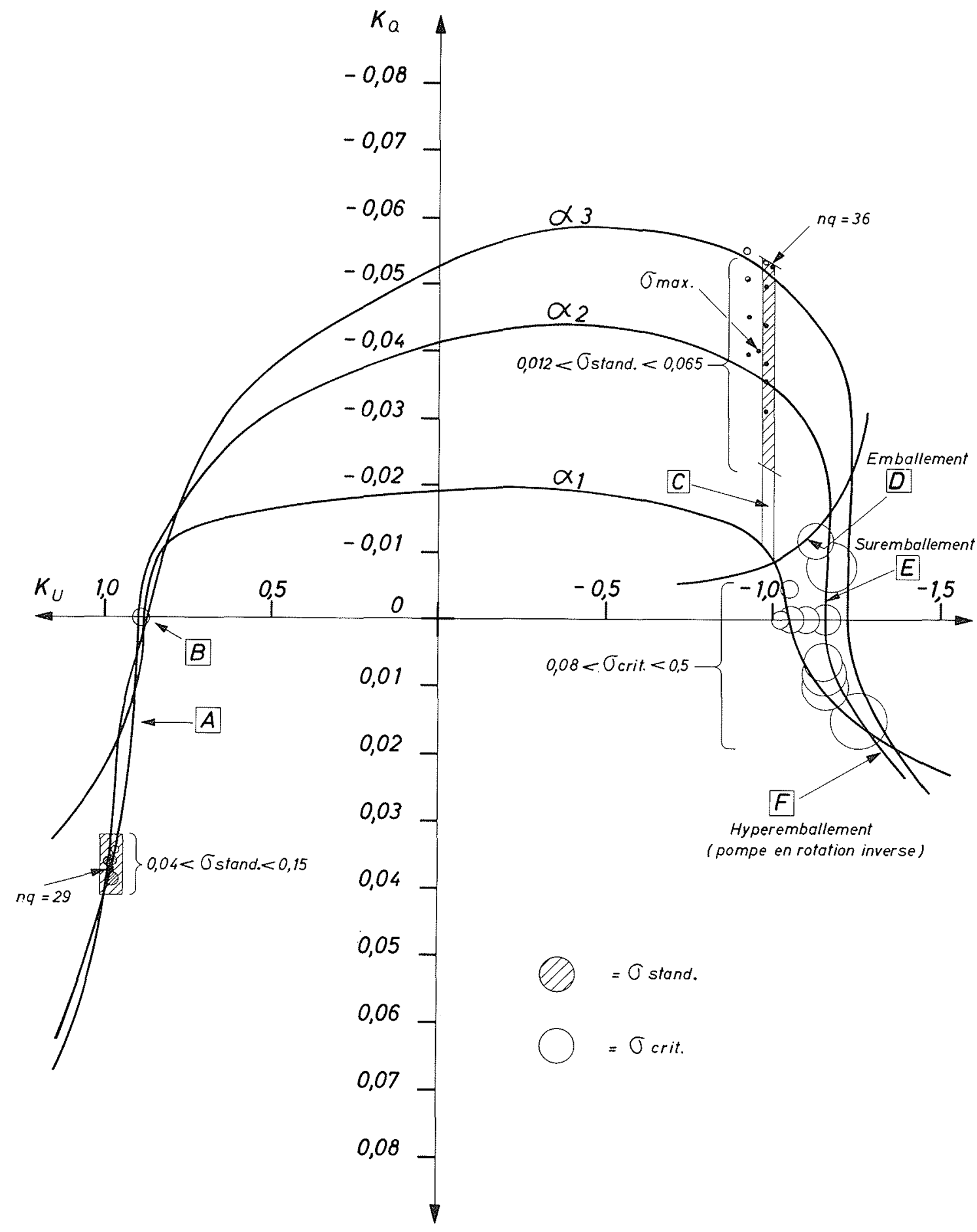

Figure 1-Caractéristiques générales d'une pompe-turbine.

successivement celles qui feront l'objet de notre étude, soit :

A) la zone d'instabilité en pompe (débits partiels);

B) le point à débit nul en pompe ;

C) la zone de fonctionnement à charges réduites en turbine ;

D) l'emballement;

E) le suremballement;

F) l'hyperemballement (ou pompe en rotation inverse).
Les zones hachurées de fonctionnement normal ne seront pas examinées dans le présent exposé.

Le fonctionnement des machines hydrauliques, lors de démarrages et d'arrêts ou plus généralement de transitoires, a pris une importance particulière ces derniers temps, jusqu'à avoir des répercussions souvent déterminantes sur la durée d'une machine réversible. On sait en effet que le nombre de démarrages et d'arrêts de ces machines est très supérieur à celui des turbines classiques et que d'autre part, 
surviennent dans beaucoup d'installations des transitoires portant la machine dans la zone de l'hyperemballement [1]. La comparaison des figures 1 et 2 concernant respectivement une réversible et une turbine, toutes deux de basse vitesse spécifique, explique en partie ce fait. En effet, la zone de fonctionnement en turbine de la première machine se situe plus près de la courbe d'emballement.

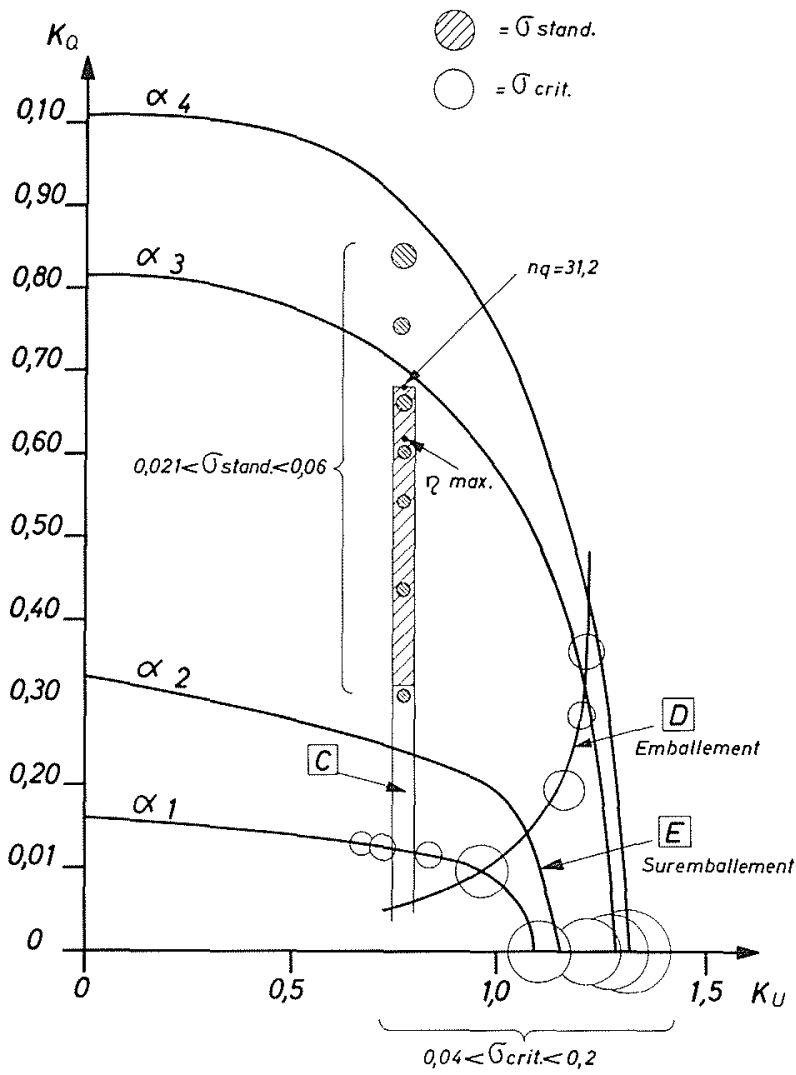

Figure 2 - Caractéristiques générales d'une turbine Francis.

Nous mettrons en évidence l'influence de la cavitation sur les caractéristiques moyennes, dans les zones précitées, en consacrant un bref chapitre à chacune d'elles. Finalement, on fera quelques considérations relatives aux caractéristiques instantanées tout en examinant quelques-unes des grandeurs qui caractérisent le fonctionnement d'une machine hydraulique et leurs variations en fonction du sigma.

\section{Influence de la cavitation sur les caractéris- tiques moyennes}

Pour avoir un tableau de l'influence de la cavitation sur les caractéristiques moyennes des turbines et des pompes-turbines, il est nécessaire de mettre en évidence, sur les diagrammes de fonctionnement des machines, les valeurs des coefficients de cavitation standards et critiques $(\sigma)$. Le sigma standard trouve sa définition précise dans les normes $C E I$ [2] à travers la chute de rendement et peut, par conséquent, être appliqué dans les champs de fonctionnement normaux de la machine, soit en pompe, soit en turbine, c'est-à-dire où le concept de rendement prend une signification. Le sigma critique remplace le sigma standard dans les champs de fonctionnement où l'on ne peut pas parler de rendement: c'est la valeur du coefficient de cavitation pour laquelle la relation entre $K_{u}, \mathrm{~K}_{q}$ et $K_{m}$ est modifiée de façon à provoquer une variation, d'une des trois grandeurs, supérieure à $2 \%$ de sa valeur nominale.

Nous nous rapporterons donc au sigma critique pour analyser l'influence de la cavitation sur le fonctionnement de la machine en pompe aux débits réduits jusqu'à zéro, en turbine aux débits fortement réduits, en emballement et en hyperemballement, c'est-à-dire aux zones qu'on a mentionnées et définies par "débits partiels".

Les diagrammes généraux de fonctionnement en examen sont ceux déjà cités de la figure 1 et de la figure 2.

Sur chaque diagramme apparaissent :

a) les courbes correspondant à certaines ouvertures en turbine et en pompe (dans le cas de la machine réversible)

b) les zones de fonctionnement normales en turbine et en pompe (dans le cas de la machine réversible); c) le point de rendement maximal en turbine et ceux nominaux de fonctionnement en turbine et en pompe avec l'indication des $n_{q}$ correspondants. Comme on le voit, les vitesses caractéristiques des deux machines sont assez proches.

Une série de petits cercles vides ou hachurés exprime respectivement les sigma critiques et standards à l'échelle adéquate : on observera comment la valeur du sigma limite, c'est-à-dire du sigma au-dessus duquel est assurée la constance dans les caractéristiques de la machine, tend à augmenter au fur et à mesure que l'on sort de la zone de rendement maximum.

On peut donc affirmer que dans les champs de fonctionnement à débit partiel une machine hydraulique de vitesse spécifique basse, telle que celles examinées, présente des valeurs de sigma critiques sensiblement supérieures à celles standards : sauf cas exceptionnels, le rapport est compris entre les valeurs 4 et 8.

Dans le champ de fonctionnements à débit(s) partiel(s), on ne peut pratiquement plus parler d'observation de cavitation, à cause des écoulements perturbés, comme dans le cas des régimes normaux. Une première observation nous paraît essentielle : pour le fonctionnement d'une machine hydraulique à débit partiel, il est nécessaire d'effectuer l'étude expérimentale du modèle avec le sigma d'installation en analogie parfaite pour obtenir des caractéristiques qui puissent être extrapolées avec certitude à la machine industrielle.

\section{Etude de la zone $A$ : instabilité en pompe}

Le diagramme de la figure 3 représente le phénomène typique d'instabilité qu'on note dans les pompesturbines à haute vitesse spécifique, quand elles fonctionnent à débit partiel. On remarquera que la cavitation influe sur l'extrémité de la branche supérieure 


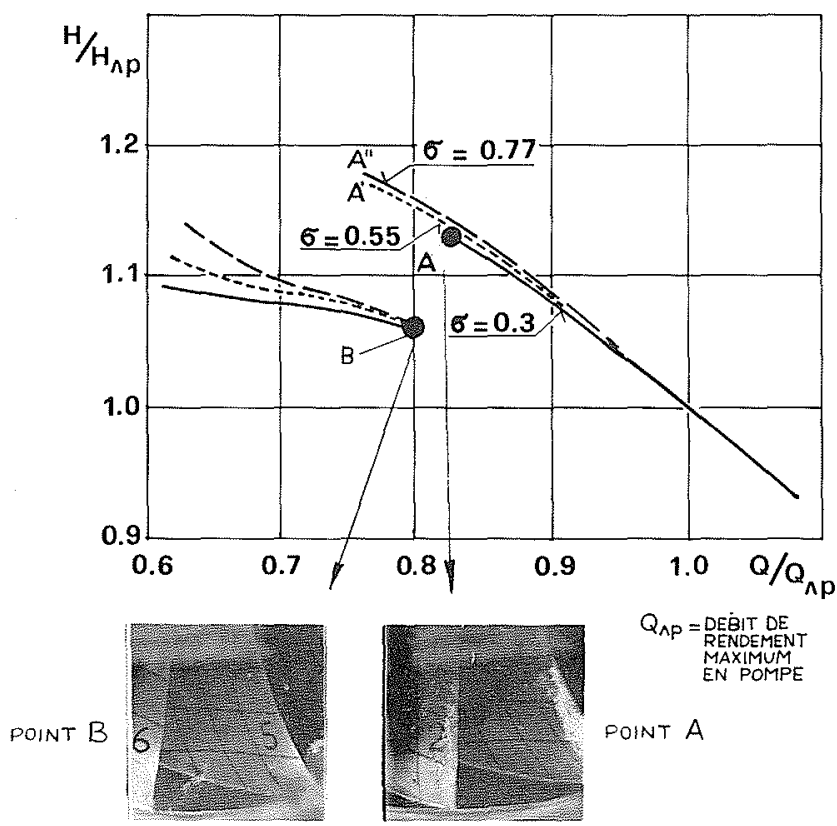

Figure 3 - Pompe-turbine $n_{q}=80$ : Fonctiohnement en pompe Analyse de l'instabilité à ouverture constante.

(du point $A$ à $A^{\prime}$ et de $A^{\prime}$ à $A^{\prime \prime}$ ) mais pas sur celle de la branche inférieure. En augmentant le sigma de 0,3 à 0,77 , le point terminal de la branche supérieure se déplace progressivement vers des débits inférieurs.

Le passage d'une branche de la caractéristique à l'autre a lieu suivant la caractéristique du circuit extérieur de la machine : en particulier, si la pompe est connectée dans un circuit ayant des pertes de charges négligeables, le chemin décrit par la machine est une ligne droite horizontale. Il est très important de remarquer qu'à débit égal, les points sur les deux branches correspondent à deux régimes toutà-fait différents, l'un tranquille - celui de la branche supérieure - l'autre perturbé et caractérisé par une recirculation de l'eau. Nos observations ont confirmé un phénomène déjà remarqué par d'autres constructeurs : la cavitation se manifeste de façon classique, c'est-à-dire adhérente à l'aube aux points de la branche supérieure, tandis qu'aux points de la branche inférieure la situation change substantiellement en donnant lieu à des sillages en sens contraire de celui de l'écoulement principal au point de jonction de la couronne, signe clair des refoulements qui révèlent une recirculation à l'intérieur de la roue.

Le phénomène décrit ci-dessus caractérise le fonctionnement d'une machine réversible en pompe [3] et en détermine très souvent le dimensionnement.

\section{Etude de la zone $B$ : point de débit nul en pompe}

En ce point de fonctionnement, la cavitation affecte principalement le coefficient de couple. Pour des valeurs de sigma supérieures à celles de l'installation, le chiffre de couple des machine étudiées, de faibles et moyennes vitesses spécifiques, accuse une augmentation non négligeable.

Ce phénomène est confirmé pour différents types
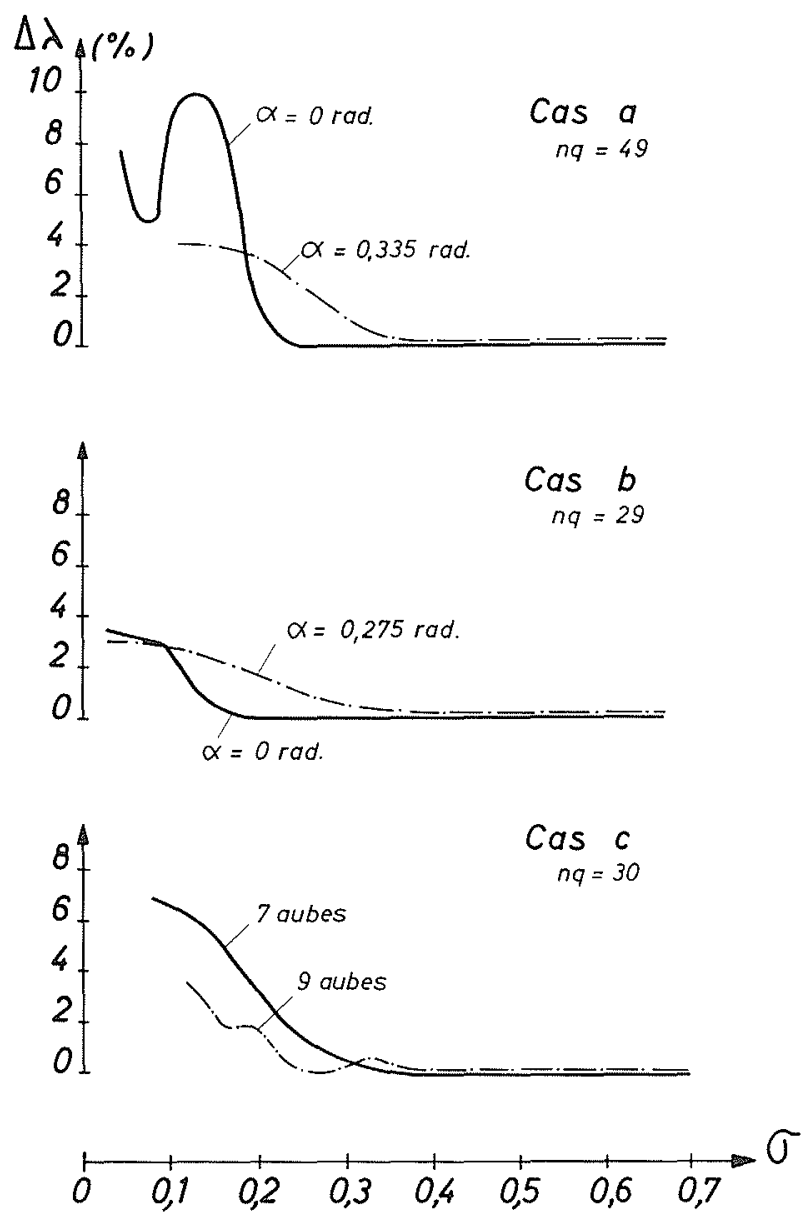

Figure 4 - Pompe-turbine : influence du $\sigma$ sur la puissance à débit zéro en pompe.

de machines avec ou sans distributeur, comme indiqué sur la figure 4 qui montre l'allure du chiffre de puissance à débit nul au fur et à mesure que varie le sigma : a) pour une réversible monoétage à moyenne vitesse spécifique $\left(n_{q \wedge}=49\right)$ pour deux différentes ouvertures du distributeur, respectivement $\alpha=0,335$ rad. et $\alpha=0$.

b) pour une réversible monoétage à basse vitesse spé-r cifique $\left(n_{q \wedge}=29\right)$ pour deux différentes ouvertures du distributeur respectivement $\alpha=0,275 \mathrm{rad}$. et $\alpha=0$.

c) pour une réversible monoétage à basse vitesse spécifique $\left(n_{q \wedge}=30\right)$ équipée successivement avec deux roues différentes.

Les essais sur modèle doivent donc tenir compte du sigma d'installation effectif, afin d'obtenir des prévisions suffisamment précises pour le dimensionnement de la machine électrique industrielle. D'autre part, il faut relever que cette transposition à la machine industrielle fait intervenir des effets d'échelle encore très mal connus.

\section{Etude de la zone $C$ : fonctionnement à débits réduits en turbine}

Il s'agit d'un sujet auquel on a dédié plusieurs travaux. Il suffit de rappeler l'ample bibliographie dé- 
rivant des travaux du WG 3 de l'AIRH constituée en 1975 à Leningrad sous la présidence de M. Elder et ayant comme thème : "Causes and dynamic effects of unsteady draft tube flows, including the problem of the air injection", et aussi les derniers travaux présentés au récent symposium AIHR de Tokyo [5], [6], [7].

Ce sujet est traité d'autre part par MM. Henry, Wegner et Graeser en cette même session.

Il peut être intéressant de signaler ici un cas d'instabilité qui a été constaté sur une turbine modèle dans une zone de fonctionnement normal et, plus précisément, dans toute une bande à débit constant correspondant à une ouverture du distributeur très proche de celle de rendement maximum.

Comme l'on voit en figure 5, aux sigma élevés on retrouve, comme dans le cas de la turbine-pompe fonctionnant en pompe aux débits fortement réduits de la figure 3 , le régime double qui, dans ce cas, vient couper la courbe de rendement traditionnelle des rendements quand le $\sigma$ diminue jusqu'à des valeurs proches de celles d'installation.

On pourrait s'étonner du fait qu'au fur et à mesure que le $\sigma$ diminue, le phénomène d'instabilité disparaisse, mais en réalité c'est justement cette diminution du $\sigma$ qui finit par stabiliser la torche dans la configuration $A$, plus favorable.

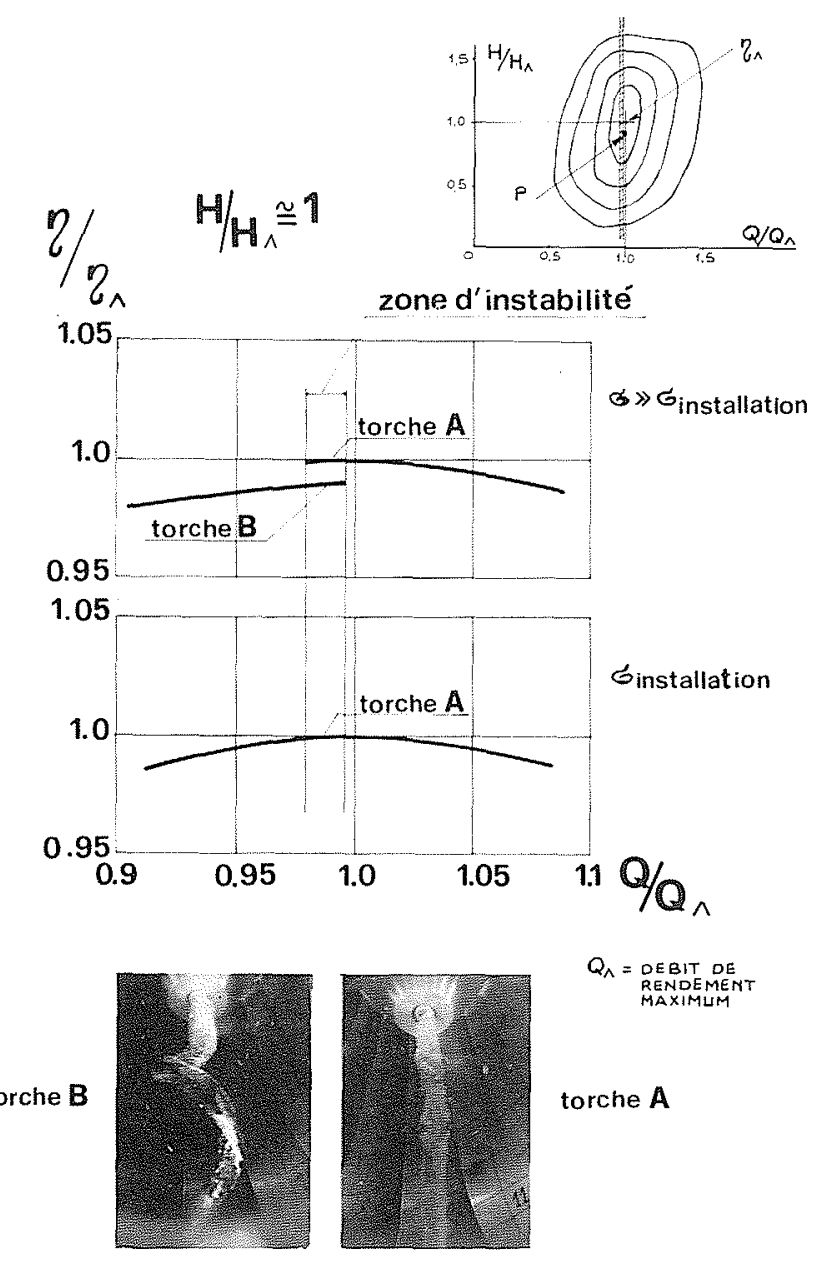

Figure 5 - Turbine Francis $n_{q}=65$ : Analyse de l'instabilité au point $P$.
Etude des zones $D$ (emballement), E (suremballement) et $F$ (hyperemballement)

Les plates-formes d'essais ne permettant pas toujours des investigations complètes à très bas sigma dans ces domaines perturbés, peu d'études ont été faites jusqu'à ce jour. Nous avons entrepris des essais sur un éventail aussi large que possible de machines, sur différents stands d'essais, pour essayer d'éclaircir ce phénomène.

En première analyse, il ressort que l'influence du sigma est réduite dans la zone de l'emballement et du suremballement pour les machines à faible et moyenne vitesse spécifique, mais qu'elle devient importante dans la zone de l'hyperemballement où elle provoque une baisse sensible des débits.

L'étude de ce phénomène prend toute son importance pour les pompes-turbines car les problèmes d'instabilité en marche à vide (pour les machines à grandes vitesses spécifiques), de même que les phénomènes transitoires inhérents à ce type de machine, sont directement affectés par cet effet.

\section{a) pompes-turbines}

La figure 6 montre les caractéristiques de fonctionnement de deux machines réversibles monoétages relativement lentes $\left(n_{q \wedge}=29\right.$ et $n_{q \wedge}=37$ ), construites et essayées par deux constructeurs différents. Pour ce type de machine, seule la zone de suremballement est influencée par la diminution du sigma. L'allure en " $S$ " des caractéristiques typiques pour les pompes-turbines lentes, subsiste même pour des sigma correspondant à ceux auxquels travaillent généralement ces machines.

Sur les pompes-turbines réversibles de vitesse spécifique élevée on peut remarquer une influence beaucoup plus marquée du phénomène. En effet, les caractéristiques confondues jusqu'à l'emballement se différencient de plus en plus, au suremballement, lorsque l'on se déplace vers l'inversion de débit. Pour les grandes ouvertures du distributeur, l'inversion de débit n'est plus possible au sigma correspondant à celui de l'installation. En effet, on observe que pour des sigma élevés, l'eau à l'aval de la roue est pratiquement exempte de bulles de cavitation, alors que pour de bas sigma, la cavitation est extrêmement violente, rendant la roue invisible et donnant lieu à l'égalisation des pressions à l'intrados et à l'extrados des aubes, ce qui rend l'inversion de débit impossible comme indiqué précédemment.

\section{b) turbine Francis}

Enfin, sur des turbines Francis à moyen $n_{q}$, on a effectué des mesures dans le quadrant turbine (Fig. 7), en faisant varier le sigma de 1,8 à 0,10 (sigma installation $=0,10$ à 0,15$)$, ceci pour une seule ouverture du distributeur, le phénomène se manifeste comme pour les pompes-turbines rapides.

Le phénomène illustré par la figure 7 est plus évident si on passe d'une turbine Francis à $n_{q}$ moyen à une turbine Francis à $n_{q}$ élevé, où les déviations que les courbes de fonctionnement, à l'emballement et surtout 

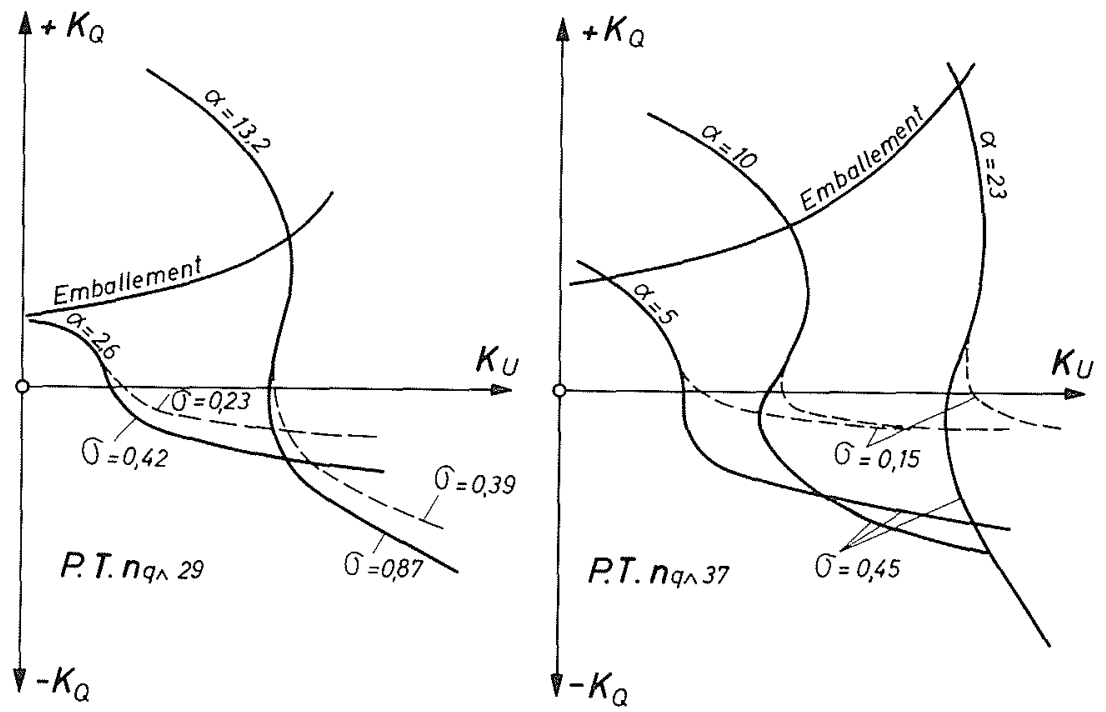

Figure 6 -Pompe-turbine : influence du $\sigma$ sur les courbes caractéristiques au-delà de l'emballement.

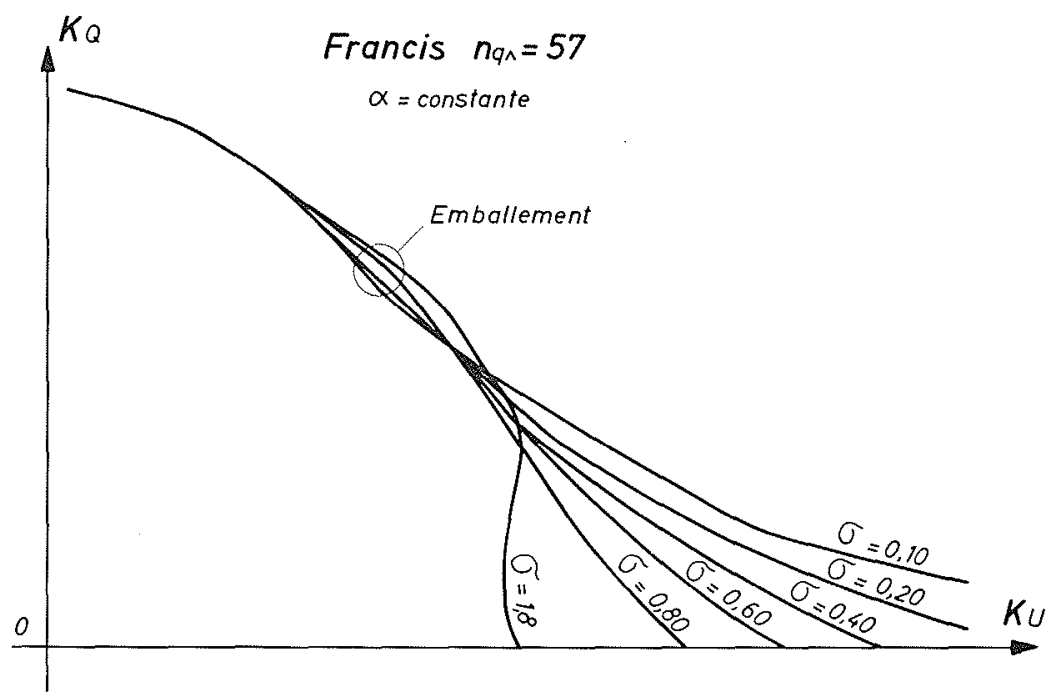

Figure 7 - Turbine Francis : influence du $\sigma$ sur les courbes caractéristiques.

au suremballement, subissent par effet de la diminution du sigma à la valeur d'installation ou à une valeur tout près de celle-ci, sont encore plus évidentes.

Des observations exposées ci-dessus, il est intéressant de souligner deux faits :

a) instabilité de la marche à vide

Le fonctionnement en marche à vide est conditionné par la pente des caractéristiques de couple $\left(K_{m}\right)$ et de débit $\left(K_{Q}\right)$.

Ces dernières pouvant être influencées par le sigma, il est important de tenir compte de ce fait lors des essais sur modèle, afin de fournir des éléments suffisamment précis sur la stabilité de la machine industrielle.

\section{b) régimes transitoires}

On a vu que les caractéristiques intéressées lors du déclenchement de machines réversibles diver- gent en général en fonction du sigma. Ce fait ne simplifie pas le calcul précis des transitoires pour la machine industrielle, car dans ces régimes, le sigma n'est pas une constante. On peut relever toutefois que, dans presque tous les cas étudiés, nous avons rencontré une tendance à l'atténuation $\mathrm{du}$ régime transitoire quand on prend en considération les courbes caractéristiques tracées au sigma d'installation. En utilisant, comme ce fut le cas jusqu'à présent, une caractéristique mesurée à haut sigma, l'on va dans le sens de la sécurité.

\section{Influence de la cavitation sur les caractéris- tiques instantanées}

Dans la partie précédente, on a examiné l'influence du coefficient de cavitation sur les caractéristiques moyennes (dans le temps) d'une machine 
hydraulique. Cette influence se vérifie aussi sur les caractéristiques instantanées. Sur une turbine Francis de vitesse caractéristique moyenne, nous avons relevé les fluctuations de quelques grandeurs significatives telles que : les pressions amont et aval de la roue et le couple à l'arbre en fonction du sigma.

Le diagramme en colline de la figure 8 montre les trois points où l'on a effectué la recherche : les diagrammes (Fig. 9, 10 et 11) montrent les résultats des mesures effectuées et qui se rapportent respectivement au point " $A$ " (machine à pleine charge), au point " $B$ " (machine à charge $4 / 10$ ) et au point " $C$ " (machine à l'emballement). Sur les diagrammes, apparaissent les mesures de pulsation de couple à l'arbre et de pression (ces dernières mesurées aux points de la turbine qui apparaissent dans les esquisses) en fonction du $\sigma_{u}$. Le champ des $\sigma_{u}$ inclut ceux correspondant au prototype. Le $\sigma_{u}$ est calculé par rapport à la cote du cône où l'on suppose que la cause principale du phénomène est localisée.

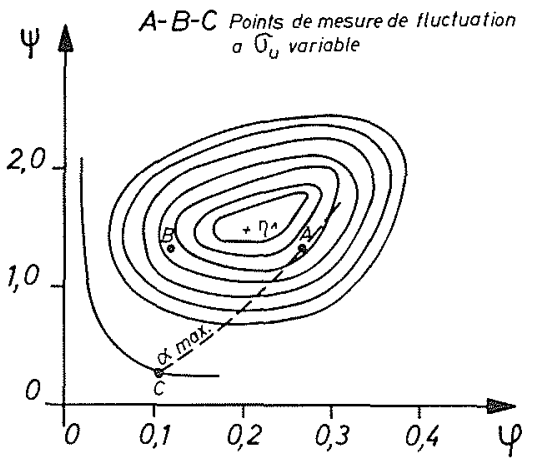

Figure 8 - Turbine Francis $n_{q \wedge}=65$ : diagramme en colline.
Sur la figure 9 pour machine à pleine charge (point $A$ ) et figure 10 pour machine à demi charge (point $B$ ), on a indiqué, outre à la valeur de crête des fluctuations lue dans les enregistrements, la racine carrée de la valeur quadratique moyenne, c'est-à-dire la valeur efficace, de l'harmonique fondamentale relevée dans les analyses du spectre. On peut remarquer que dans les pulsations de pression sous roue, qui apparaissent dans le diagramme de la figure 10, le rapport entre la valeur de crête et la valeur efficace est très proche de celle théorique 1,41 qui s'obtient quand le signal est une sinusoide pure.

Dans le cas du point d'emballement $C$, le spectre a un contenu d'harmoniques presque continu; il n'est par conséquent pas possible de déterminer l'harmonique prédominante comme dans les cas précédents. (fig. 11)

Quelques observations sont possibles:

a) au point de charge $A$, les courbes mesurées ne dénoncent pratiquement aucune variation de fluctuation, ni des pressions, ni du couple, même quand le coefficient de cavitation se réduit à des valeurs très proches de celles d'installation;

b) au point $B$ à charge $4 / 10$, les pulsations tendent à augmenter quand le coefficient de cavitation tend vers des valeurs proches de celles d'installation : pour les valeurs inférieures les pulsations, aussi bien de couple que de pression, se réduisent ;

c) au point d'emballement maximum (point $C$ ) les pulsations de pression atteignent un maximum pour des sigmas supérieurs à celui présumable d'installation, tandis que les fluctuations de couple demeurent plus ou moins constantes par rapport aux variations du sigma.

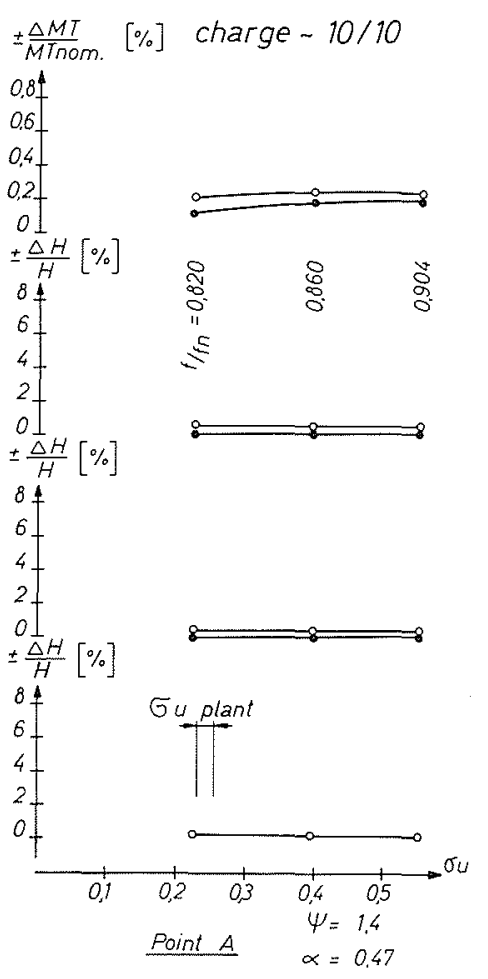

Figure 9 - Fluctuations de pression et du couple dans le point $A$ de figure 8.

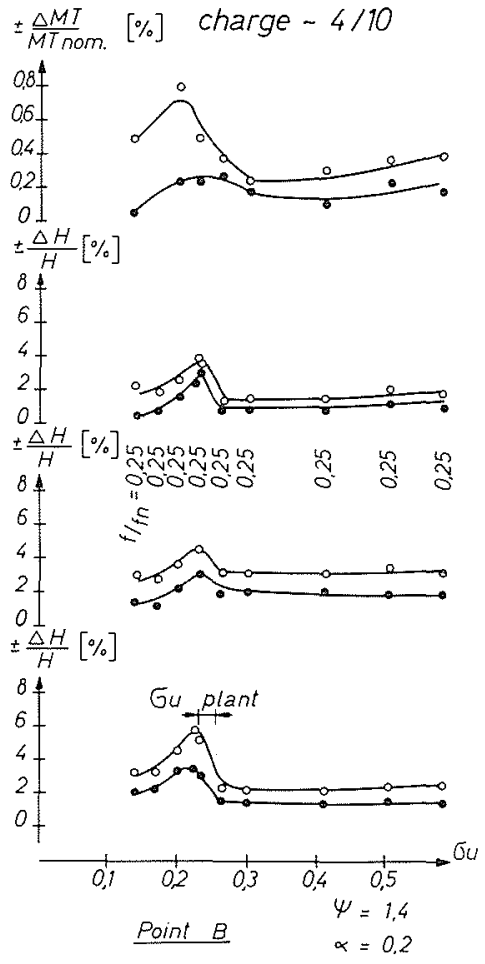

Figure 10 - Fluctuations de pression et du couple dans le point $B$ de figure 8 .

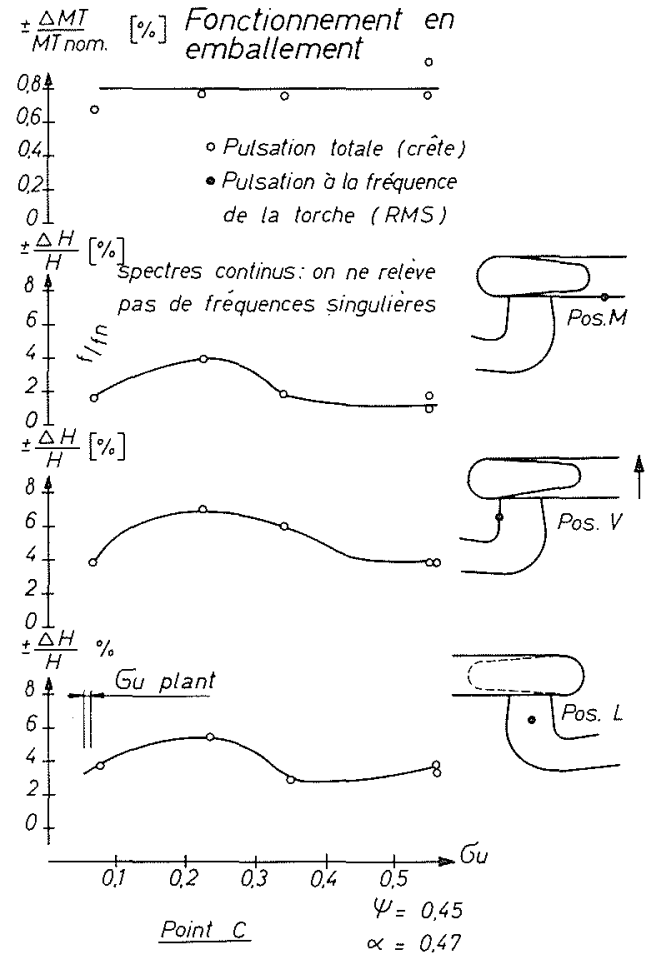

Figure 11 - Fluctuations de pression et du couple dans le point $C$ de figure 8 . 
En ce qui concerne les fréquences fondamentales de pulsation, tandis que celles à charge $4 / 10$ sont pratiquement constantes par rapport aux variations du sigma, celles à pleine charge tendent à augmenter avec le sigma jusqu'à atteindre des valeurs très proches de la fréquence de rotation aux sigma élevé.

Des essais analogues faits sur une turbine modèle Francis à vitesse caractéristique plus élevée $\left(n_{q \wedge}=\right.$ 90) ont substantiellement confirmé les résultats exposés aux points $a$ ), $b$ ) et $c$ ) précédents, avec la seule exception des valeurs de pulsation de pression, qui se révélèrent largement supérieures à celles mesurées sur la turbine Francis modèle à vitesse spécifique moyenne, quand la machine est à l'emballement.

Sur les deux machines, les pulsations maximales aussi bien de pression que de couple se vérifient en correspondance d'un coefficient de cavitation très près de celui d'installation, ce qui doit être dûment considéré par le constructeur.

Les mesures de fluctuation, dont on a parlé, ont été faites sur des machines modèle dont l'aspirateur débouchait dans une cuve ayant une très grande section dans laquelle on n'a relevé aucune fluctuation de pression; mais puisqu'en amont de la machine un dispositif de ce type n'existait pas, on peut penser que les mesures effectuées - et en particulier celles réalisées aux débits réduits - peuvent être influencées par les caractéristiques d'impédance du circuit d'essai [8]. Par conséquent, il faut être très prudent dans la transposition de ces phénomènes au prototype.

\section{Conclusions}

De la revue rapide, obligatoirement incomplète, que l'on vient de faire, il ressort que l'influence du sigma est sensible, non seulement dans les zones de fonctionnement normal d'une machine hydraulique (soit en turbine, soit en pompe), mais aussi dans toutes les autres zones examinées, qui peuvent être définies comme zones de fonctionnement anormal ou, si l'on veut, à débit partiel.

Cette influence détermine d'un côté la modification des caractéristiques moyennes, de l'autre, tend dans certains cas à aggraver les caractéristiques instantanées. Il est donc essentiel pour le dimensionnement correct d'une machine hydraulique, que tout le champ de fonctionnement du modèle soit expérimenté en laboratoire dans un champ de sigma qui inclut ceux prévus sur l'installation : seulement de cette façon, il sera possible d'avoir les éléments pour prévoir avec bonne approximation le comportement de la machine industrielle dans toutes les conditions possibles de fonctionnement.

II est à souhaiter que les expériences futures de laboratoire et industrielles servent à définir avec une précision toujours plus grande l'influence d'un facteur, tel que le coefficient de cavitation, dont l'importance aux effets du bon comportement de la machine apparaît déterminante.

\section{Remerciements}

Les auteurs désirent remercier M. Guiton, M. Martin (Neyrpic) et M. le Prof. Henry, Directeur du Laboratoire de machines hydrauliques de l'EPUL de Lausanne, pour la précieuse contribution fournie.

\section{Bibliographie}

[1] BOUSSUGES, JARRIAND, AMBLARD. - Analyse des forces pulsatoires agissant sur les parties tournantes des turbines-pompes. La Houille Blanche $n^{\circ} 1 / 2,1980$.

[2] CEI, Publication 193A. Premier complètement à la Publication 193, 1965.

[3] FISHER, WEBB. - Effect of cavitation on the discontinuity point and on alternating pressures and gate torques on a pump/turbine model in the pump cycle. Joint Symposium ASCE - AIRH - ASME, Fort Collins, 1978.

[4] BORCIANI, ROSSI, ZANETTI. - Multistage reversible machine operation at zero discharge in pumping operation. $10^{e}$ Symposium AIRH, Tokyo 1980.

[5] GREIN. - Vibration phenomena in Francis turbines : their causes and prevention. $10^{e}$ Symposium AIRH, Tokyo 1980.

[6] FISHER, PALDE, ULITH. - Comparison of draft tube surging of homologous scale model and prototype Francis turbines. $10^{e}$ Symposium AIRH, Tokyo 1980.

[7] NISHI, KUBOTA, MATSUNAGA, SENOO - Study of swirl flow and surge in an elbow type draft tube. $10^{e}$ Symposium AIRH, Tokyo 1980.

[8] FANELLI. - Problemi d'instabilità del sistema idraulico. Convegno AEI-FAST, Milano 22/11/79.

Pour compléter mon exposé, je pense qu'il est opportun de projeter deux diapositives. La première (diagramme 4RS 15144 ci-joint) montre en synthèse l'allure des courbes d'emballement en fonction du coefficient de cavitation pour trois Francis à basse, moyenne et haute vitesse spécifique, ainsi que l'allure de la vitesse d'emballement dans une Kaplan. On remarquera que si pour la Francis à basse vitesse spécifique l'influence du sigma sur la vitesse maximum d'emballement est pratiquement nulle, elle devient sensible quand la vitesse spécifique de la machine augmente : en particulier, sur le modèle d'une turbine Francis ayant une vitesse spécifique $n_{q}=90$ on a une diminution du coefficient de vitesses périphérique $k_{u}$ très près de $5 \%$ quand on passe de valeurs du sigma très élevées à des valeurs près du sigma d'installation. Pour les Kaplans, au contraire, il se produit un phénomène inverse et, $\mathrm{du}$ moment que la vitesse maximum d'emballement est fonction non seulement du sigma, mais aussi de l'ouvertures des aubes, les essais d'emballement sur une turbine Kaplan sont d'habitude plutôt complexes.

La deuxième diapositive, qu'on vient de projeter (voir diagramme 4RS 15148 ci-joint), illustre l'examen de l'influence du coefficient de cavitation sur les caractéristiques dynamiques d'une turbine Francis par les résultats des mesures effectuées sur une machine avec vitesse spécifique différente de celle considérée à la figure $8 \mathrm{du}$ mémoire, c'est-à-dire avec un $n_{q}$ de $95 \mathrm{au}$ lieu de 65 . 


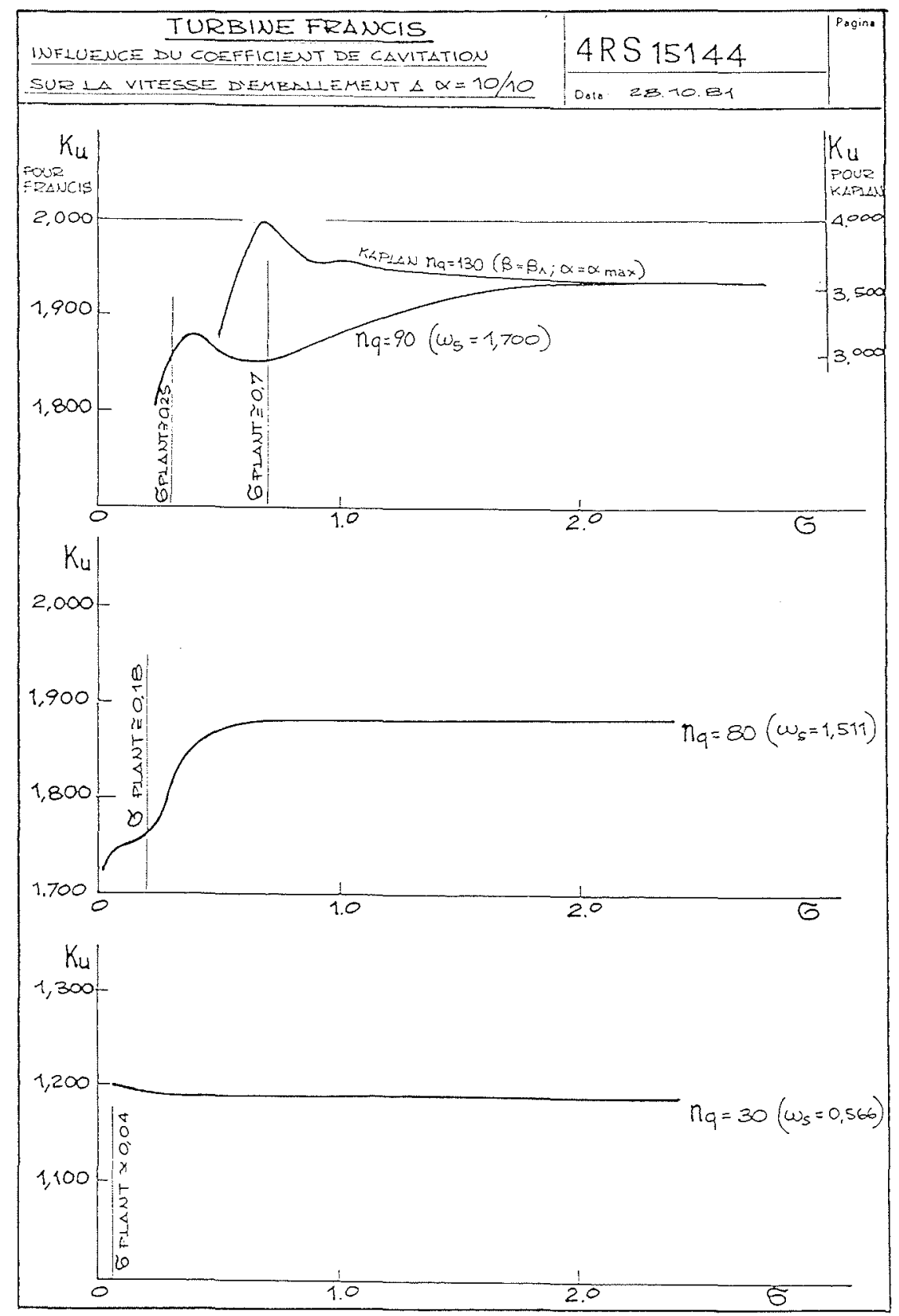

La diapositive 4RS 15148 qu'on est en train de projeter montre les fluctuations des grandeurs indiquées dans les figures 9,10 et 11 pour une machine à $n_{q}=95$ fonctionnant à charge $5 / 10$ et en plus, celles du débit et de la chute : les courbes qui représentent la pulsation à la fréquence de la torche (crête), peuvent être comparées à celles de la figure 10 qui se réfèrent au point $B$. On remarquera qu'au sigma "plant" il y a, comme dans le cas de la Francis à $n_{q}=65$, une pointe dans les fluctuations de pression et de couple à l'arbre. Mais l'observation la plus importante dérive de la comparaison des courbes à trait continu avec celles hachurées: les courbes à trait continu se rapportent à la machine en version normale, c'est-à-dire avec coude de décharge de type traditionnel, celle hachurées se rapportent à la machine avec tuyau d'aspiration droit.
On pourra remarquer, alors que les deux courbes ont pratiquement la même allure en ce qui concerne les pulsations de pression en aval du T4, c'est-à-dire immédiatement sous roue, à l'intrados, que la machine avec tuyau d'aspiration droit donne lieu, en amont, à des pulsations de pression de chute, de débit et de couple à l'axe qui sont sensiblement inférieures. Nous pensons que ce phénomène, qui à notre avis est fondamental, peut être expliqué sur la base des considérations qui suivent. D'autre part, on peut explicuer l'anomalie apparente dans les fluctuations de pression à la sortie de la roue à l'extrados (point T2) par la symétrie existant dans le cas du tuyau d'aspiration droit et la symétrie conséquente dans le comportement des pressions, dans ce cas T2 et T4 (comparer les courbes hachurées).

Comme on le sait, il y a une composante tangen- 


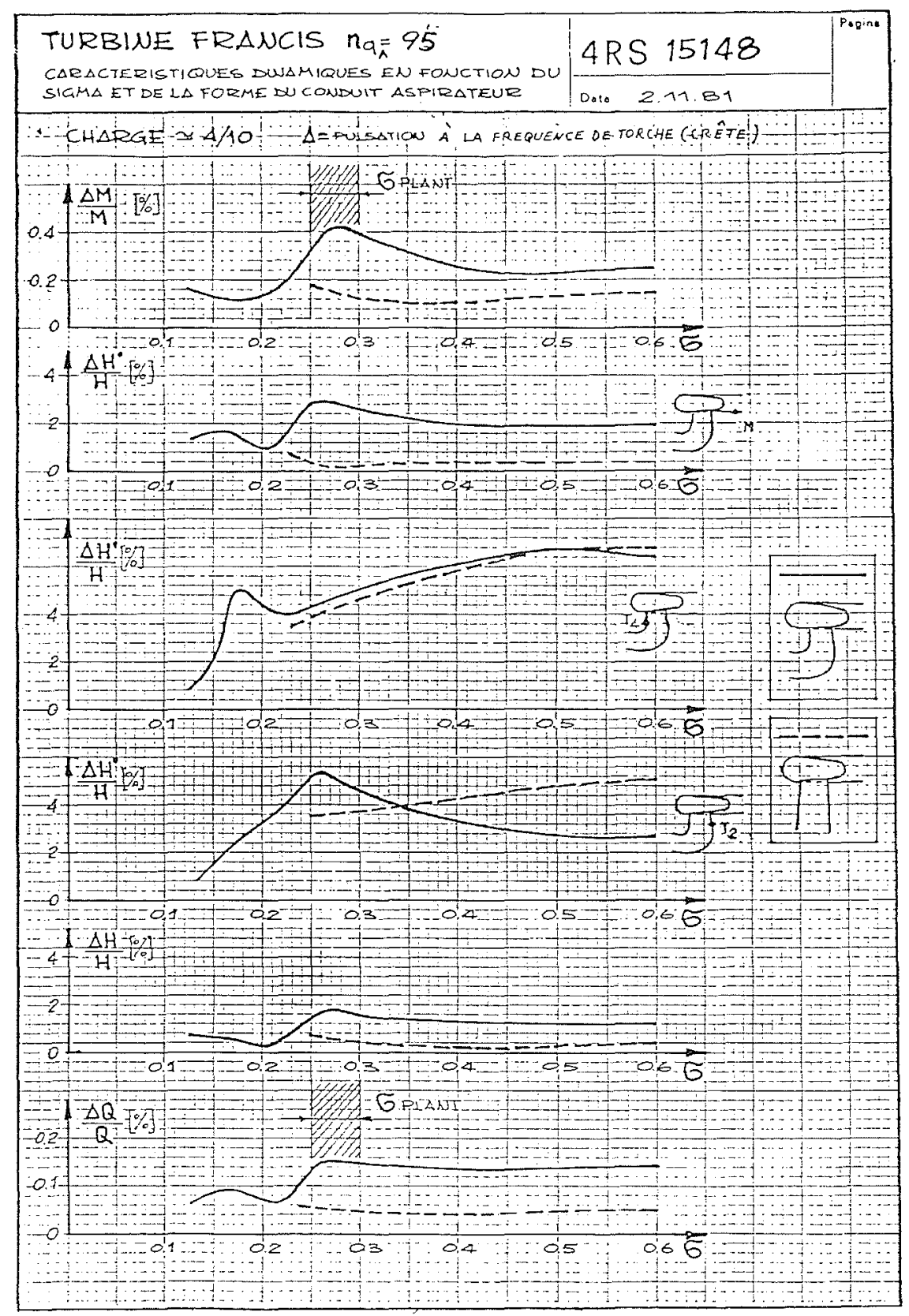

tielle considérable à la décharge de la roue Francis aux débits partiels qui provoque la formation d'une torche. En examinant la caractéristique de cette torche, on remarque que la rotation de son axe est excentrique, dans la section considérée, suivant la fréquence bien connue de rotation de Rheingans qui a une valeur de $0,25 / 0,30$ par rapport à celle de rotation de la machine.

Dans le cas d'un tuyau d'aspiration droit, la torche à la décharge de la roue n'a pratiquement pour effet que de faire fluctuer la pression sous roue, mesurée dans un point quelconque de la périphérie, alors que la valeur moyenne des pulsations sur toute la circonférence reste constante. Voilà pourquuoi, comme il résulte de la diapositive, les fluctuations de pression en aval de la roue, à l'intrados, ne diffèrent pas dans les deux cas examinés, c'est-à-dire avec le tuyau d'aspiration droit, ou coudé.

On peut donc affirmer que, soit avec tuyau d'aspiration droit, soit avec tuyau d'aspiration coudé, le phénomène de la torche ne donne pas lieu à des changements substantiels dans les fluctuations de pression à la décharge de la roue. Au contraire, la situation change nettement si l'on examine les pulsations des autres caractéristiques de la machine, c'est-à-dire pressions en amont, chute et couple qui sont très modestes en cas de tuyau d'aspiration droit. Les valeurs de ces pulsations deviennent sensibles si l'on introduit un coude tel que celui normalement adopté pour les turbines Francis : on peut schématiser ce phénomène en supposant que le coude remplit la fonction d'un étranglement périodique, probablement lié au brusque changement de direction $\left(90^{\circ}\right)$ de la torche. Il en résulte un effet qui peut être schématisé dans une perte de charge qui varie suivant la fréquence de la torche et par conséquent détermine la variation du débit transitant dans la machine, la pulsation de couple agissant sur l'arbre et la pulsation de la chute. 


\section{Discussion}

Président : J. CHEVALIER

Le président remercie M. BORCIANI.

A la suite des interventions de MM. ROTH, KECK, GRAESER et OSTERWALDER, des précisions sur les conditions expérimentales et les méthodes de mesure sont fournies par MM. BORCIANI, ROSSI et ZANETTI.

Le point à $10 / 10$ de charge n'est pas rigoureusement défini, il est arbitraire mais la vitesse spécifique est calculée au point de rendement maximal. L'axe du distributeur a été pris comme référence pour le calcul de o. Il eût été préférable de prendre l'endroit exact où se produit le phénomène que l'on veut étudier comme c'est le cas lorsqu'on étudie des figures de cavitation sur modèle.

Les fluctuations de débit ont été mesurées par comparaison électrique des signaux fournis par deux transducteurs đifférentiels de pression situé à 2 mètres l'un de l'autre sur la conduite droite à section constante en amont de la turbine. Ce système convient pour les basses fréquences (environ jusqu'au double de la fréquence de la torche).
La variation de $\sigma$ a été réalisée à chute constante.

M. WEGNER constate que les résultats présentés par $M$. BORCIANI sont très proches de ceux qu'il présente avec MM GRAESER et HENRY et souligne l'intérêt d'avoir supprimé le coude de l'aspiration pour les essais. Il précise qu'il a observé, comme M. BORCIANI, que la première fréquence du spectre des pressions à 1'aspiration reste bien dominante lorsque le $\sigma$ diminue. Toutefois, la contribution de la deuxième fréquence du spectre qui reste faible en ce qui concerne les pulsations de pression devient prépondérante en ce qui concerne les fluctuations de couple.

Il $y$ a ainsi une démonstration empirique du fait que la première fréquence représente un phénomène de fluctuation locale (hélice) tandis que la deuxième fréquence représente un phénomène de pulsation globale (passage complexe du vortex dans l'aspirateur).

M. ROSSI précise que la suppression du coude de l'aspirateur a apporté un gain d'environ $1,5 \%$ sur le rendement.

\section{Abstract \\ Influence of cavitation on average and instantaneous characteristics of turbines and pump turbines.}

The influence of cavitation on the average characteristics of a Francis turbine or of a Francis type pump-turbine is studied beyond the normal operating range examining the following areas: (a) instability area during operation as a pump; (b) zero flow pump mode; (c) area of reduced flow as a turbine; (d) runaway speed; (e) turbine-brake; (f) reverse pump quadrant.

This study, which has been carried out on the basis of laboratory measurements on model, clearly shows that the critical sigma values found in the 5 areas mentioned above are higher than those of standard sigma values for the areas of the machine in normal operating conditions.

Particulat importance is placed on two observed cases of hydraulic instability, the first is a pump-turbine operating as a partial flow pump, the other is a turbine of average specific speeed : instability is a function of the cavitation coefficient which determines the shift from one hydraulic behaviour to another at runner outlet.

The influence of cavitation on the instantaneaus charac teristics is examined in a turbine at full load, at a low rate of flow and at runaway speed, referring particularly to pressure pulse and to shaft torque pulse: also, in this case, the decrese in cavitation coefficient towards values corresponding to those in plant operation causes an increase of the fluctuations both at partial flow and at runaway speed for some of the magnitudes examined.

To conclude, when designing a hydraulic machine, the laboratory check on the model should be extended to a wide range of sigma values covering the values projected for the prototype so as to extend the prototype expectations in operation to all the ranges involved both in steady state and transient conditions. 